\title{
ОПРЕДЕЛЕНИЕ КРИТИЧЕСКОЙ СКОРОСТИ ГРУЗОВОГО ВАГОНА НА ТЕЛЕЖКАХ ТИПА Ү25 ПУТЕМ КОМПЬЮТЕРНОГО МОДЕЛИРОВАНИЯ
}

\section{DETERMINATION OF CRITICAL RATE FREIGHT WAGONS ON A TROLLEY Y25 BY TYPE OF COMPUTER SIMULATION}

\author{
Представил д-р техн. наук, профессор И.Э. Мартынов
}

\begin{abstract}
По нормативным документам, которые регламентируют процедуры допуска к эксплуатации грузовых вагонов колеи 1520 мм по показателям их динамических качеств, как изготовленные, так и модернизированные вагоны должны подвергаться испытаниям почти одинакового объема. По отношению к вагонам с модернизированными ходовыми частями такой подход нельзя считать рациональным.

Согласно европейским нормам применяются два метода измерения величин, на основании которых оцениваются динамические качества подвижного состава: нормальный и упрощенный. При нормальном методе для оценки обеспеченности безопасности движения рельсовых экипажей нового типа измеряют силы взаимодействия колес и рельсов (боковая $Y$ и вертикальная $Q$ составляющие). Кроме того, измеряются боковые и вертикальные ускорения кузова над тележками, а также боковые ускорения на концах рам тележек.
\end{abstract}

При упрощенном методе, который применяется в случаях, когда испытаниям подвергаются модифицированные вагоны, или когда изменяются условия их эксплуатации, ограничиваются измерением только боковых сил $H$ между колесной парой и рамой тележки, т.е. буксовых сил (вместо сил взаимодействия колес и рельсов) или ускорения кузова и ходовых частей в горизонтальном и вертикальном направлениях.

Идея заключается в измерении и регистрации ускорений кузова (пятников) вагона. По экспериментальным данным, полученным в узких диапазонах скоростей движения, уточняется базовая имитационная математическая модель, и далее, путем компьютерного моделирования, определяется весь спектр нормативных показателей, необходимых для полной оценки ходовых динамических качеств исследуемого вагона. В отличие от полномасштабных полигонных ходовых динамических испытаний подвижного состава, которые проводятся, как правило, 
на специально отведенных участках железнодорожного пути с фиксированными характеристиками и техническим состоянием, испытания в условиях эксплуатации проводятся на разнородных по конструкции и состоянию участках пути. Полученная при этом информация дает достоверное и достаточно полное представление о динамических характеристиках вагонов в реальных условиях эксплуатации.

В рамках методики проведение натурных испытаний ориентировано на получение статистически достаточных оценок ускорений пятников, как величин наиболее чувствительных к условиям взаимодействия подвижного состава и пути, а также к режимам движения поездов. При опытных поездках запись динамических процессов осуществляется с привязкой к плану пути, скорости и режиму движения поезда.
Так, использование тележки типа Y25 на территории стран СНГ в системе интермодальных и интероперабельных перевозок является достаточно перспективным. Поэтому для расчетов была выбрана фитинговая платформа для большегрузных контейнеров модели 13-7024.

Для исследования динамических качеств создана компьютерная модель вагона на тележках Ү25. Объект «Вагон» состоит из одного тела «Кузов» и двух включённых подсистем «Тележка», которая образована телом «Рама», двумя подсистемами «Колесная пара с буксами», двумя телами «Скользун», четырьмя телами «Стакан опорный» и четырьмя телами «Толкатель». Связь между элементами подсистемы «Тележка» представлена восемью билинейными пружинами буксового подвешивания, четырьмя пружинами скользуна, восемью держателями [1] (рис. 1).

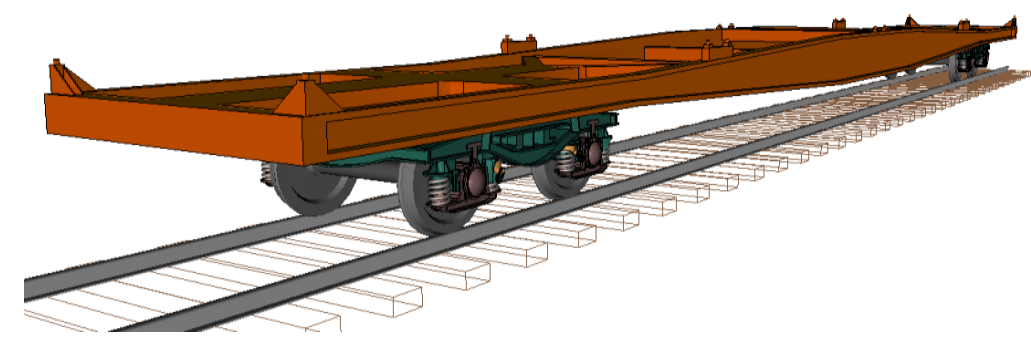

Рис. 1. Общий вид модели вагона

Так было проведено моделирование динамики вагона по упрощенной методике. На рис. 2-9 приведены графики зави- симостей CKO (RMS) исходных величин от скорости движения вагона $[2,3]$.

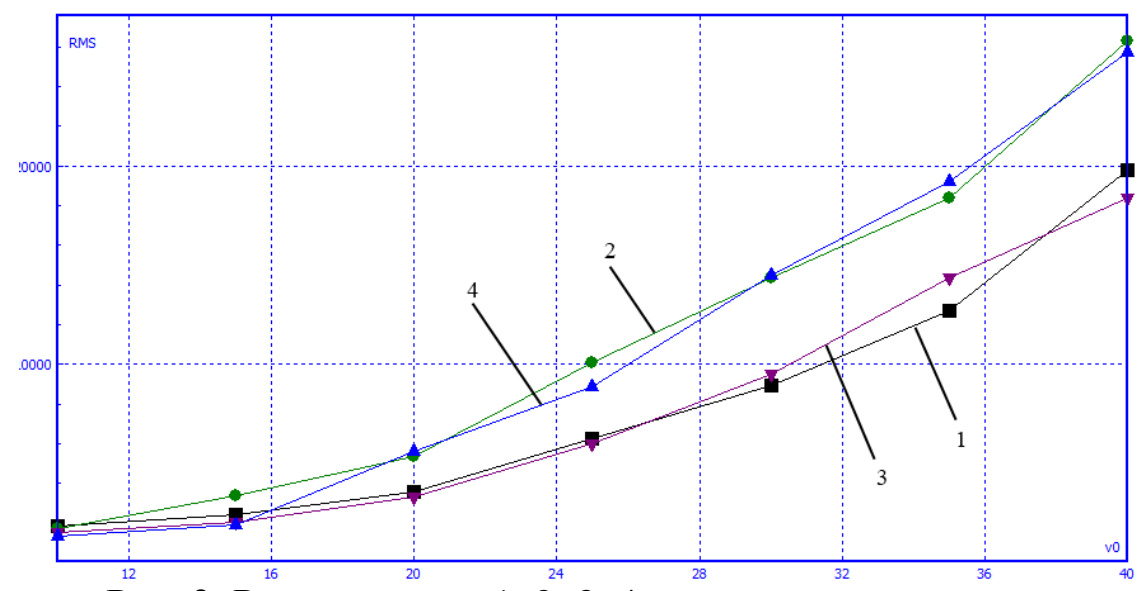

Рис. 2. Рамные силы: 1, 2, 3, 4 - номера колесных пар 
Из графика видно, что гранично допустимые рамные силы достигаются при $V=21 \mathrm{~m} / \mathrm{c}$.

Уровень сил взаимодействия колес и рельсов на путях колеи 1520 мм не нормируется. Поэтому приходится ограничиваться качественной оценкой зависимостей, графики которых приведены на рис. 3 и 4.

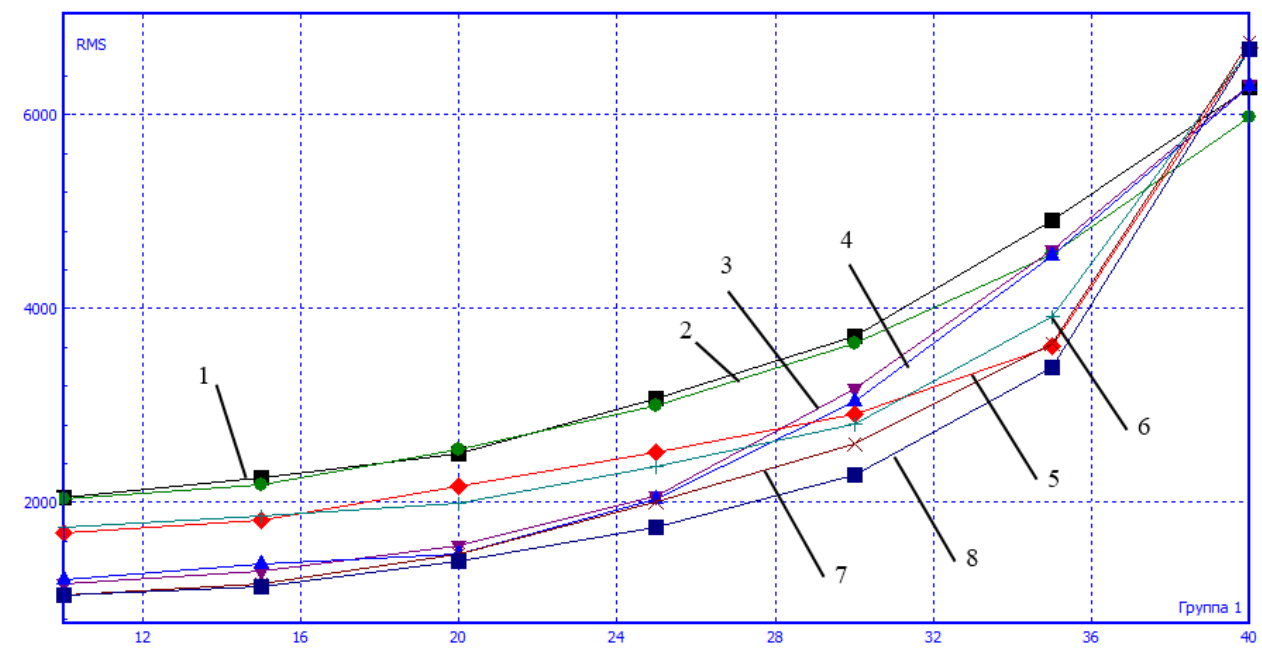

Рис. 3. Горизонтальные силы взаимодействия колес и рельсов: $1 \ldots 8$ - номера колес

Из графика видно, что большим уровнем боковых сил отличается первая колесная пара до скорости движения $35 \mathrm{~m} / \mathrm{c}$.

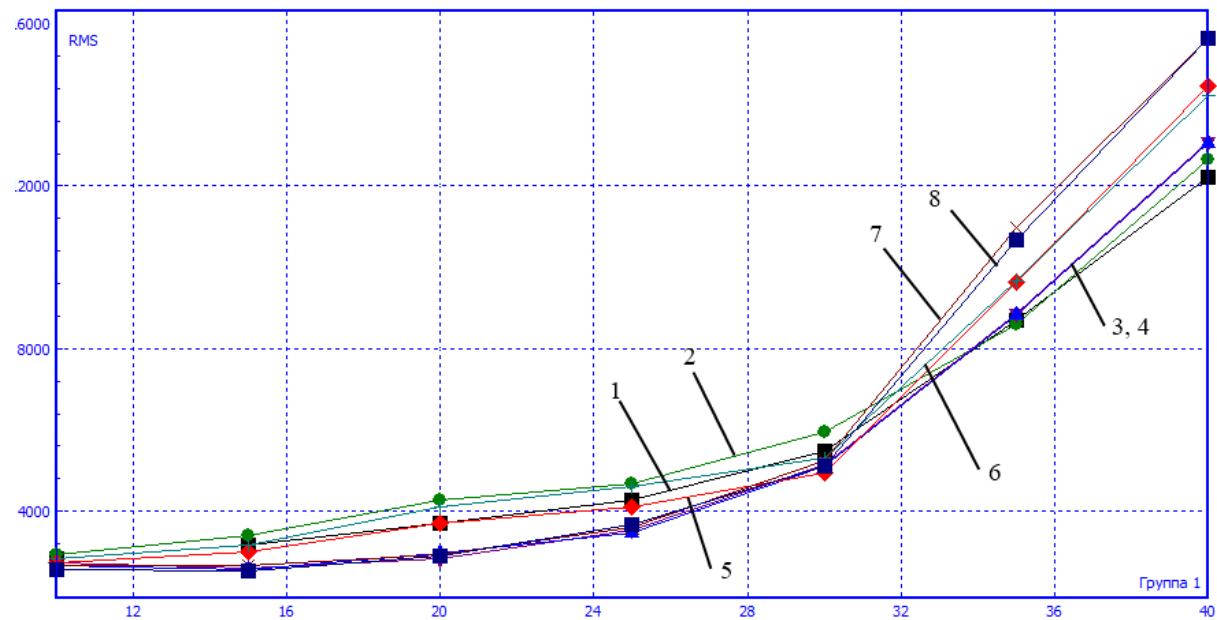

Рис. 4. Вертикальные силы взаимодействия колес и рельсов: $1 \ldots 8$ - номера колес

Вертикальные силы взаимодействия можно оценить как отличные в скоростном диапазоне до 35 м/с включительно. Тем не менее, наблюдается ускорение темпа нарастания этих сил с увеличением скорости движения после $30 \mathrm{~m} / \mathrm{c}$. 


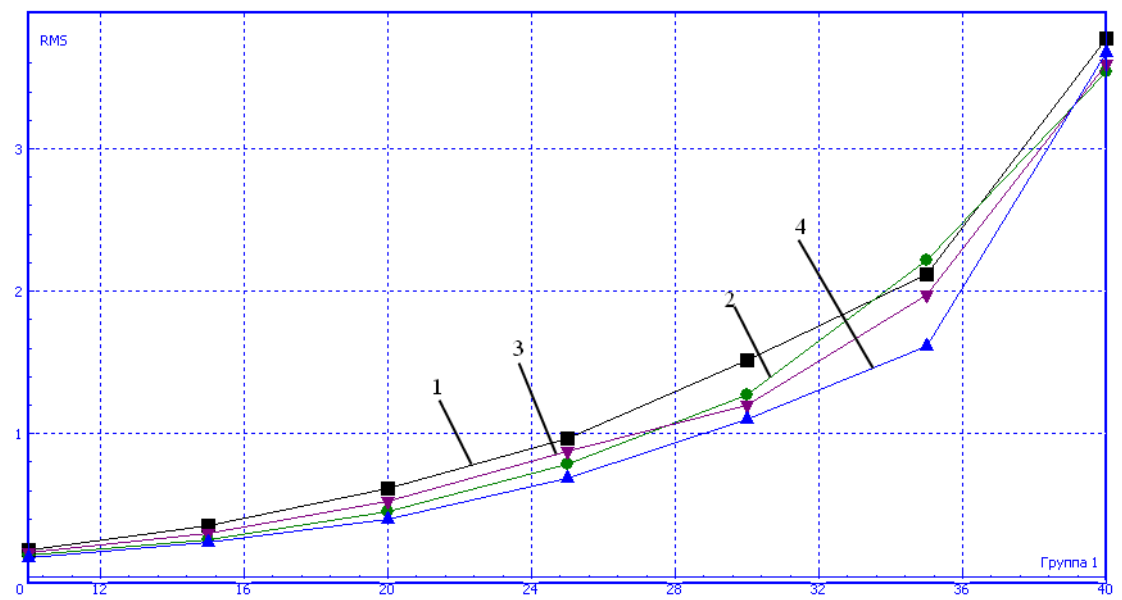

Рис. 5. Горизонтальные ускорения колесных пар: 1, 2, 3, 4 - номера колесных пар

По рассчитанным горизонтальным ускорениям колесных пар предельно допустимая скорость составляет около $32 \mathrm{M} / \mathrm{c}$.

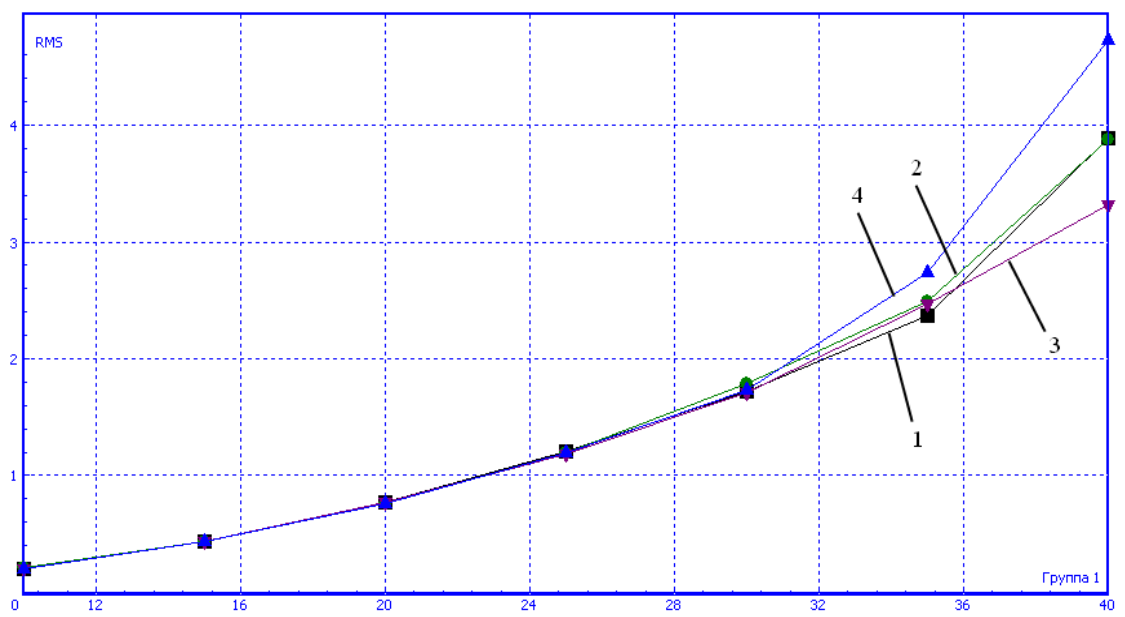

Рис. 6. Вертикальные ускорения колесных пар: 1, 2, 3, 4 - номера колесных пар А по вертикальным ускорениями - 36 м/с.

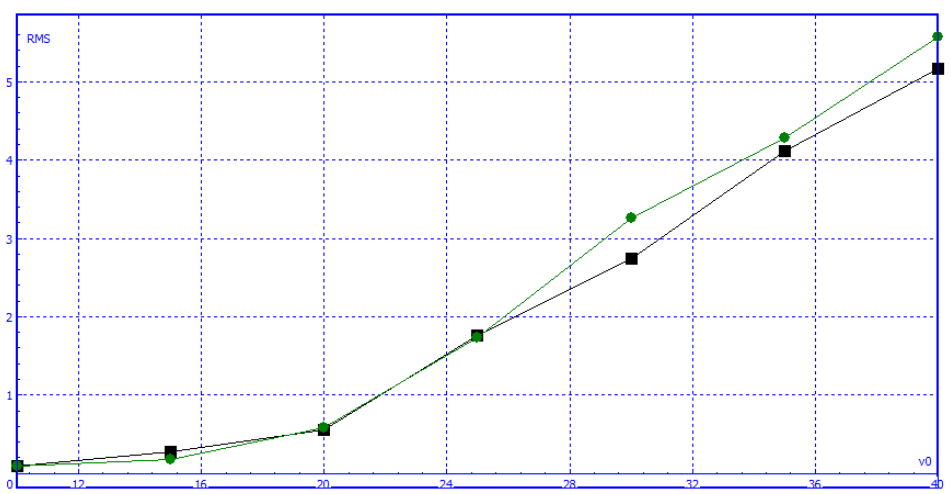

Рис. 7. Горизонтальные ускорения пятников (черная линия - первая тележка, зеленая вторая) 
Интенсивность подъема уровня допустимая скорость движения вагона ускорений заметно увеличивается при составляет $24 \mathrm{~m} / \mathrm{c}$. $\mathrm{V}>20$ м/с. Причем по уровню ускорений

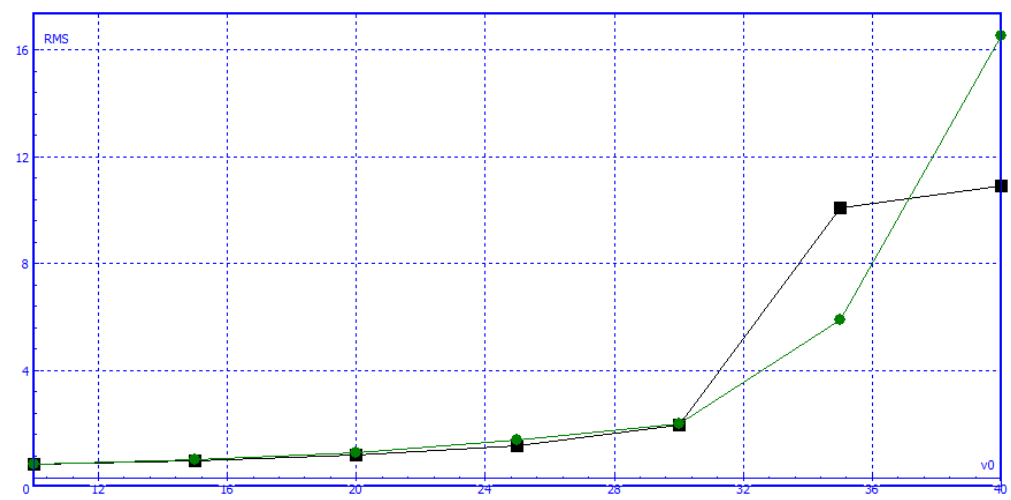

Рис. 8. Вертикальные ускорения пятников (черная линия - первая тележка, зеленая - вторая)

Уровень вертикальных ускорений пятников остается допустимым при скорости движения $30 \mathrm{~m} / \mathrm{c}$.

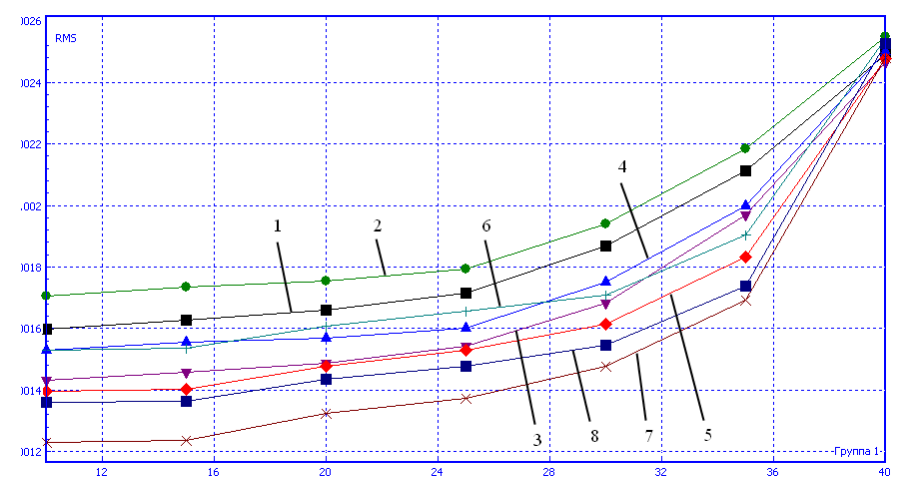

Рис. 9. Углы набегания колес на рельсы: $1 \ldots 8$ - номера колес

Величины углов набегания колес на рельсы, как и силы взаимодействия, также не нормируются на путях колеи 1520 мм. Как видно из графика, крупнейшими углами набегания отличаются колеса направляющей колесной пары.

За счет проведения анализа и оценки полученных путем моделирования динамических величин определялась критическая скорость вагона.

В этом исследовании критическая скорость порожнего грузового вагона на тележках типа Y25 составила 21 м/с (76,5 км/ч).
Эксплуатация тележек типа Y25 на путях колеи 1520 мм возможна при определении рациональных параметров рессорного подвешивания и устройств для опирания кузова вагона на тележки.

Разработанная компьютерная модель позволяет исследовать вынужденные пространственные колебания грузового вагона, варьировать основные геометрические и инерционные параметры, параметры пружинного подвешивания, а также параметры, характеризующие износ элементов ходовой части на базе полной параметризации модели. 


\section{Список литературы}

1. Черняк, Г.Ю. Побудова комп'ютерної моделі європейського вантажного візка типу Y25 в програмному комплексі "Универсальный механизм" [Текст] / Г.Ю. Черняк, А.А. Стецько // Зб. наук. праць. - Харків: УкрДАЗТ, 2011. - Вип. 123. - С. 147-152.

2. Нормы для расчёта и проектирования новых и модернизируемых железнодорожных транспортеров общего назначения колеи 1520 мм [Текст]. - М.: ВНИИВ-ВНИИЖТ, 1988. $136 \mathrm{c}$.

3. UIC Code 518. Testing and approval of railway vehicles from the point of view of their dynamic behaviour - Safety - Track fatigue - Ride quality.

Ключевые слова: безперегрузочные перевозки, тележка типа Y25, раздвижные колёсные пары, моделирование движения.

\section{Аннотации}

Наведено результати моделювання руху вантажного вагона на візках типу Ү25 по шляхах колії 1520 мм в програмному комплексі "Универсальный механизм".

Приведены результаты моделирования движения грузового вагона на тележках типа Y25 по путям колеи 1520 мм в программном комплексе "Универсальный механизм".

This paper presents the results of modeling the movement of freight cars at Y25 bogies type of path of $1520 \mathrm{~mm}$ in the software package "Universal Mechanism". 